\title{
Novel heterozygous compound TRMT5 mutations associated with combined oxidative phosphorylation deficiency 26 in a Chinese family: a case report
}

Shuiyan $\mathrm{Wu}^{1 \dagger}$, Weixi $\mathrm{Li}^{2 \dagger}$, Zhenjiang Bai ${ }^{1 \dagger}$, Saihu Huang ${ }^{1}$, Daoping Yang ${ }^{3}$, Hongmei Chen $^{1}$, Ying $\mathrm{Li}^{1}$, Ying Liu ${ }^{2^{*}}$ and Haitao LV ${ }^{3^{*}}$

\begin{abstract}
Background: Combined oxidative phosphorylation deficiency 26 (COXPD26) is an autosomal recessive disorder characterized by early onset, developmental delay, gastrointestinal dysfunction, shortness of breath, exercise intolerance, hypotonia and muscle weakness, neuropathy, and spastic diplegia. This disease is considered to be caused by compound heterozygous mutations in the TRMT5 gene.
\end{abstract}

Case presentation: In this study, we report a female child with COXPD26 manifesting as shortness of breath, gastrointestinal dysmotility, severe developmental delay, muscle hypotonia and weakness, exercise intolerance, renal and hepatic defects, and recurrent seizures with spastic diplegia. Interestingly, the hepatic feature was first observed in a COXPD26 patient. Medical exome sequencing with high coverage depth was employed to identify potential genetic variants in the patient. Novel compound heterozygous mutations of the TRMT5 gene were detected, which were c.881A > C (p.E294A) from her mother and c.1218G >C (p.Q406H) and c.1481C>T (p.T494M) from her father.

Conclusion: The newly emerged clinical features and mutations of this patient provide useful information for further exploration of genotype-phenotype correlations in COXPD26.

Keywords: COXPD26, Medical exome sequencing, TRMT5, Mutation, Case report

\section{Background}

Combined oxidative phosphorylation deficiency 26 (COXPD26; MIM \#616539), which includes developmental delay, shortness of breath, exercise intolerance, spasticity hyporeflexia, and hypotonia leading to the patient being unable to sit, stand, or walk without support, and

\footnotetext{
*Correspondence: d201077400@alumni.hust.edu.cn; haitaolvsoochow1@163. com; haitaosz@163.com

†Shuiyan Wu, Weixi Li and Zhenjiang Bai contributed equally to this work.

${ }^{2}$ Institute of Pediatric Research, Children's Hospital of Soochow University, Suzhou, Jiangsu Province 215025, P.R. China

${ }^{3}$ Department of Cardiology, Children's Hospital of Soochow University,

Suzhou, Jiangsu Province 215025, P.R. China

Full list of author information is available at the end of the article
}

decreased mitochondrial complex activity, is an autosomal recessive disorder characterized by a highly variable phenotype [1-3]. COXPD26 was first characterized in 1989 by Haller et al. [1]. To date, only four COXPD26 cases from three unrelated families are described [1-3]. Powell et al. reported a woman (the same case Haller et al. reported) and a 7-year-old boy, both of whom showed muscle weakness, exercise intolerance, neuropathy, gastrointestinal problems, spasticity with hyperreflexia, increased serum lactate levels, and decreased activity of mitochondrial complex IV. Over the following years, the woman developed exocrine insufficiency with malabsorption, liver cirrhosis, and renal tubulopathy. 
The boy showed additional features, including earlyonset growth retardation, abnormal facial features, and hypertrophic nonobstructive cardiomyopathy. Mark reported two additional adult patients, both of whom had disease onset in childhood [2]. The patients presented similar aspects of the previously described phenotypes, including muscle weakness, exercise intolerance, neuropathy, and spastic diplegia [1-3]. Prematurity and the global developmental delay of one case were similar to the symptoms of the 7-year-old boy. Shortness of breath upon exertion was observed in Patient 1, as well as in the previously reported woman [2] (Table 1).

TRMT5 (tRNA (guanine(37)-N1)-methyltransferase 5, NM_020810) is considered as the gene causing COXPD26 [1, 2]. TRMT5 is located on chromosome $14 \mathrm{q} 23.1$ and encodes a 509 -amino-acid protein that is produced in the cytosol and then transported into the mitochondria [2]. In mitochondria, it aids in the modification of tRNA 1-methylguanosine $\left(\mathrm{m}^{1} \mathrm{G}\right)$ at position 37 , which is next to the $3^{\prime}$ end of the anticodon [4], both in vivo and in vitro [2]. Moreover, TRMT5 is not very specific with respect to the structure of bound tRNA which will be methylated [5]. In eukaryotes, about 11 kinds of tRNA can be modified with $\mathrm{m}^{1} \mathrm{G} 37[6,7]$. The methylation helps in reading frame maintenance by protecting against frameshifting when the peptides are elongated on the mRNA. Otherwise, $a+1$ frameshift error may occur, causing premature termination of protein synthesis [8]. It is conceivable that defective production of essential proteins due to errors such as frameshifts will disturb cellular function. For example, disruption of the TRMT5 homologous gene TrmD in Salmonella typhimurium, Streptococcus pneumoniae, and Saccharomyces cerevisiae severely impairs microorganismal growth $[9,10]$. In humans, when TRMT5 is transported into mitochondria, it methylates the tRNAs that are encoded by mitochondrial DNA (mtDNA) [11]. Altered tRNA methylation in mitochondria will affect the expression of proteins encoded by mtDNA and disturb respiratory function $[1$, 2]. This may be the pathogenic mechanism of COXPD26 caused by recessively inherited mutations in TRMT5. In this paper the first Chinese family with COXPD26 is described. We report a female patient with clinical features similar to previous cases of COXPD26 and a novel compound heterozygous mutation of TRMT5. The present study expands the currently available evidence concerning mutations associated with COXPD26.

\section{Case presentation}

The pedigree underwent careful physical examination. The proband's parents (I:1, I:2) were nonconsanguineous and both appeared normal. The proband (II:1) had frequently suffered from pneumonia since she was born, accompanied with shortness of breath much of the time (Fig. 1A). As a neonate, she presented laryngeal stridor and was diagnosed with laryngomalacia. She began to experience nausea and vomiting after being fed when she was 2 months old, and a gastrostomy tube was placed at that time. She presented considerable tympanites and gastrointestinal dysmotility with frequent vomiting. When she was 6 months old, she could raise her head. She could sit at the age of 10 months and stand at the age of 2 years. Developmental delay was obvious at the age of 4 years, when she reached a height of only $98 \mathrm{~cm}(<2 \mathrm{SD})$. Unfortunately, the patient suffered from motor retardation. Furthermore, she was unable to sit or stand without help, let alone walk. Moreover, muscular hypotonia and

Table 1 Genetic and clinical features in individuals up to date

\begin{tabular}{|c|c|c|c|c|c|c|}
\hline ID & Sex & TRMT5 Variations & Age at onset & Clinical course & Other features & References \\
\hline 1 & female & $\begin{array}{l}\text { C. } 312 \_315 \mathrm{del} ; \\
\text { c. } 872 \mathrm{G}>\mathrm{A}\end{array}$ & childhood & Died at age of 55 years & $\begin{array}{l}\text { life-long exercise intolerance, dyspnea, lactic acidosis, gastro- } \\
\text { intestinal issue with malabsorption, glucose intolerance, and } \\
\text { pancreatic disorder, peripheral neuropathy, muscle weakness, } \\
\text { renal tubulopathy, liver cirrhosis }\end{array}$ & {$[1]$} \\
\hline 2 & male & $\begin{array}{l}\text { C. } 312 \_315 \mathrm{del} ; \\
\text { C. } 1156 \mathrm{~A}>\mathrm{G}\end{array}$ & birth & $\begin{array}{l}\text { Alive, } \\
7 \text { years old }\end{array}$ & $\begin{array}{l}\text { premature delivery, growth retardation, gastrointestinal issue } \\
\text { with intestinal pseudo-obstruction and poor feeding, cardio- } \\
\text { myopathy, muscle hypotonia and weakness, demyelinating } \\
\text { neuropathy, global development delay, lactic acidosis }\end{array}$ & {$[1]$} \\
\hline 3 & female & $\begin{array}{l}\text { c. } 312 \_315 \mathrm{del} \\
\text { c. } 872 \mathrm{G}>\mathrm{A}\end{array}$ & childhood & $\begin{array}{l}\text { Alive, } \\
46 \text { years old }\end{array}$ & $\begin{array}{l}\text { life-long exercise intolerance, muscle weakness, spasticity, } \\
\text { axonal sensory neuropathy }\end{array}$ & {$[2]$} \\
\hline 4 & female & $\begin{array}{l}\text { c. } 312 \_315 \mathrm{del} \\
\text { c. } 872 \mathrm{G}>\mathrm{A}\end{array}$ & childhood & $\begin{array}{l}\text { Alive, } \\
51 \text { years old }\end{array}$ & $\begin{array}{l}\text { premature, global developmental delay, spasticity, progressive } \\
\text { visual loss, frequent urinary } \\
\text { tract infections, seizures }\end{array}$ & {$[2]$} \\
\hline 5 & female & $\begin{array}{l}\text { C. } 881 A>C, \\
C .1218 G>C \text { and } \\
\text { C. } 1481 C>T\end{array}$ & birth & Died at age of 5 years & $\begin{array}{l}\text { short of breath, gastrointestinal dysmotility, severe devel- } \\
\text { opmental delay, muscle hypotonia and weakness, exercise } \\
\text { intolerance, renal and hepatic defect, recurrent seizures with } \\
\text { spastic diplegia }\end{array}$ & This study \\
\hline
\end{tabular}


A
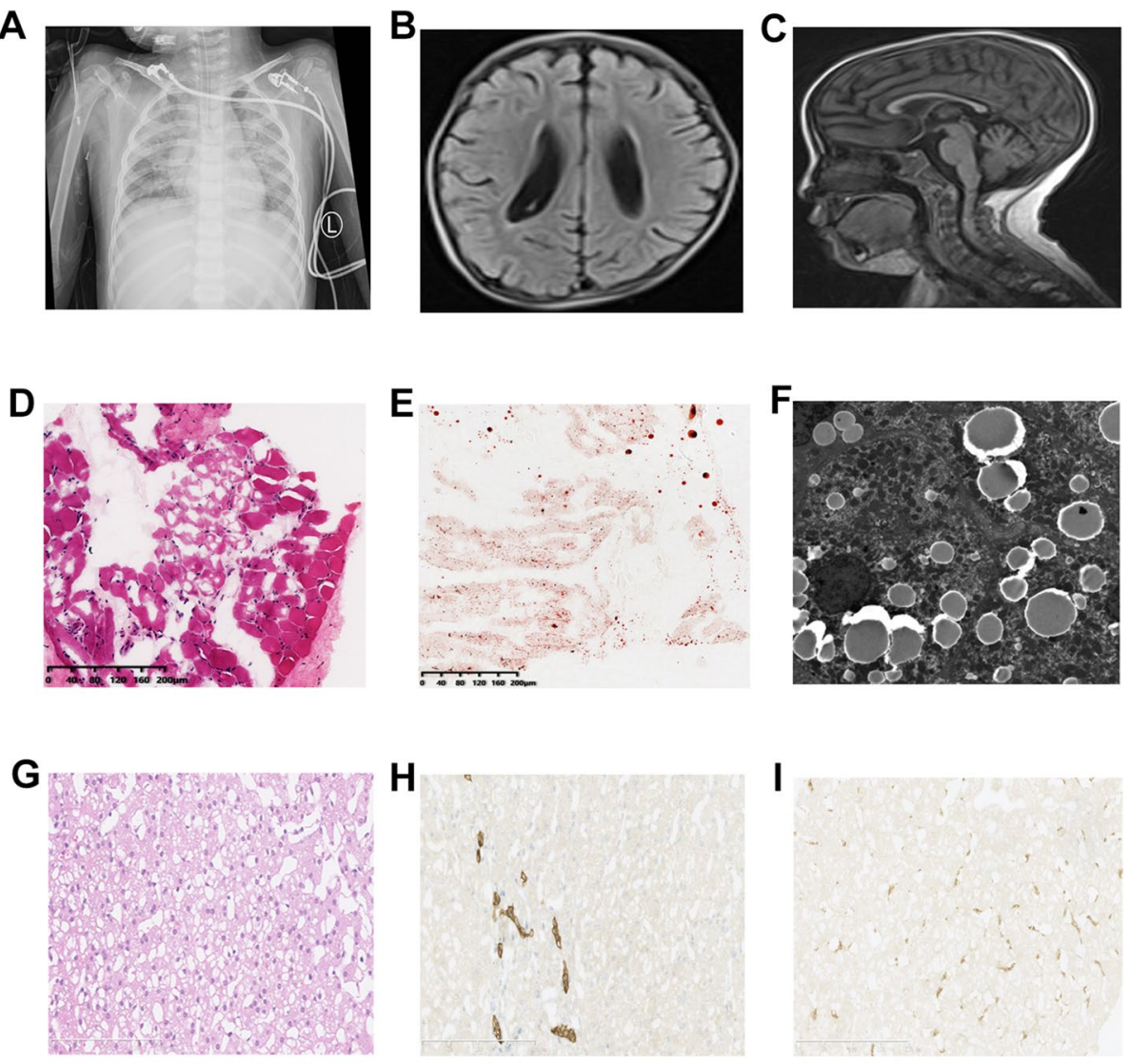

$\mathbf{H}$
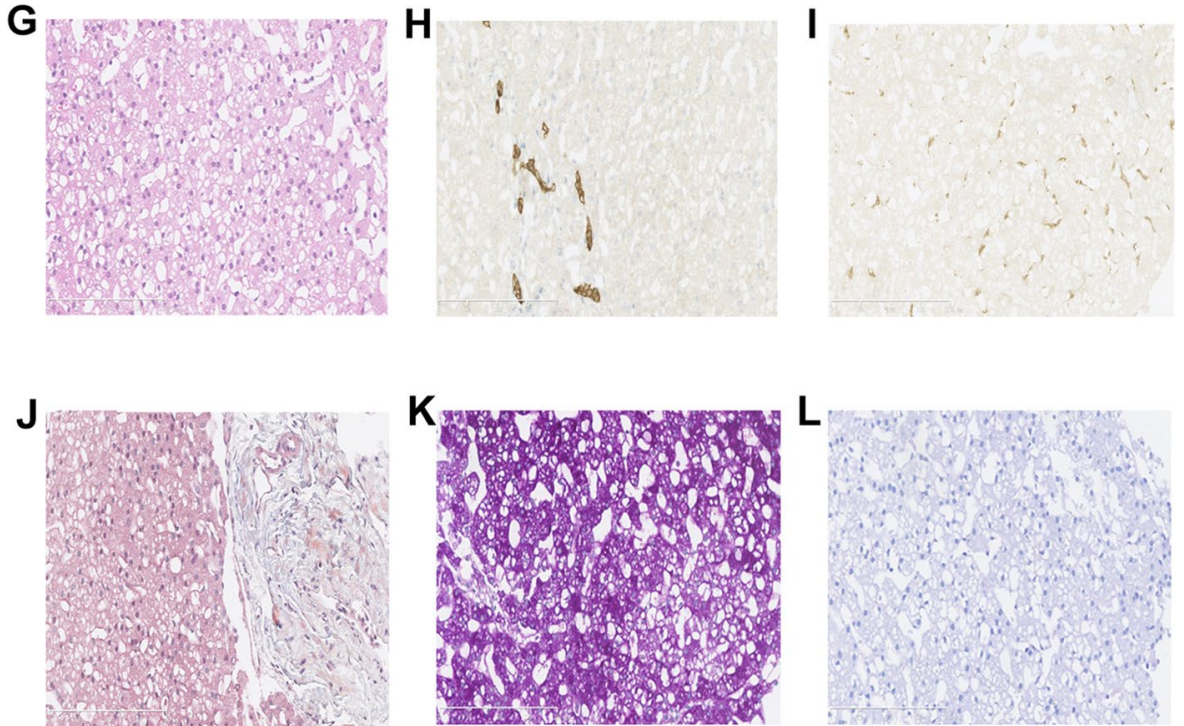

\section{$\mathbf{L}$}

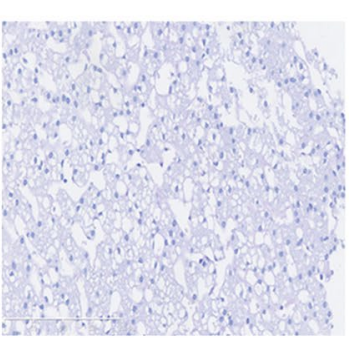

Fig. 1 The clinical and histopathological examination. A Pneumonia indicated by X-ray result. B and C Abnormality in the brain with wider extracerebral space and thinner corpus callosum. D Myopathic changes with different size, small diameter of muscle fibers were observed by HE staining and $\mathbf{E}$ increased lipid droplets by oil staining (10x). $\mathbf{F}$ Hepatic biopsy showed abundant lipid droplets by electron microscopy. G Diffuse hepatocellular steatosis was observed in liver through light microscope; $\mathbf{H}$ Immunohistochemistry showed that the bile duct epithelium was positive for cytokeratin 19; I a few activated Kupffer cells were positive for CD68; J Masson staining showed fibrous hyperplasia in the portal area; $\mathbf{K}$ PAS reaction in liver was positive due to intracytoplasmic accumulation of glycogen; L D-PAS was negative in liver. All figures of liver by light microscope were showed with $20 x$

exercise intolerance was evident. Her motor development, speech, and social adaptation were assessed to be 2.5 years delayed. She only could communicate with her family by facial expressions, gestures, and simple sounds even though she had normal visual and auditory abilities. Recurrent seizures appeared with spastic diplegia at 5 years of age. A prominent fast wave in the frontal lobe was observed by electroencephalogram examination. Brain magnetic resonance imaging (MRI) showed a wide extracerebral space (Fig. 1B) and thin corpus callosum (Fig. 1C). Muscle biopsy showed myopathic changes with different size and small diameter of muscle fibers (Fig. 1D), and increased lipid droplets (Fig. 1E). Additionally, hepatic biopsy followed by 
electron microscopy revealed abundant lipid droplets (Fig. 1F). Through light microscopy, diffuse hepatocellular steatosis was observed (Fig. 1G). Immunohistochemistry showed that the bile duct epithelium was positive for cytokeratin 19 (Fig. 1H) and a few activated Kupffer cells were positive for CD68 (Fig. 1I), and Masson staining revealed fibrous hyperplasia in the portal area (Fig. 1J). Due to intracytoplasmic accumulation of glycogen, the periodic acid-Schiff (PAS) reaction was positive (Fig. $1 \mathrm{~K}$ ) and the D-PAS reaction was negative (Fig. 1L). In addition, blood tests indicated mildly elevated alanine aminotransferase levels and a physical exam did not indicate hepatomegaly. There was an apparent renal defect with oliguria and elevated creatinine, urea nitrogen, and uric acid levels, so dialysis was employed. Inherited metabolic disorders were screened, and slightly elevated lactate levels in blood and urine were observed. Serum antibody tests for toxoplasma, rubella virus, cytomegalovirus, and herpes simplex virus were negative and the patient's plasma ammonia levels, triglyceride levels, blood glucose levels, karyotype, auditory brainstem responses, and thyroid function were all normal. There was no cardiac involvement apart from tachycardia; cardiac troponin I and creatine kinase isoenzyme levels were also in the normal range (Table 2). The patient died from stroke and respiratory failure at the age of 5 years. According to the patient's main symptoms, which were in accordance with the common features of COXPD26, a diagnosis of COXPD26 was made.

This study was conducted in accordance with the Declaration of Helsinki and was approved by the ethics committee (Children's Hospital of Soochow University, No. 2019LW010). Written consent was obtained from the parents before they participated in the genetic investigation. To achieve an accurate genetic diagnosis, medical exome sequencing was carried out with a trio sample strategy. The advantages and experimental procedures of this technique were previously described by our team [12]. Novel heterozygous missense mutations in TRMT5 (NM_020810) were detected in the proband (Fig. 2), which were c.881A $>C$ (p.E294A) from her mother and c.1218G >C (p.Q406H) and c.1481C >T (p.T494M) on the same chromosome from her father. The proband inherited these missense mutations in TRMT5, which made herself compound heterozygous. The c.881A $>\mathrm{C}$ (p.E294A) and c.1481C >T (p.T494M) mutations were reported with a minor-allele frequency of 0.0003 and 0.0008 , respectively, in the Aggregation Consortium (ExAC) Browser. The c.1218G $>\mathrm{C}$ (p.Q406H) mutation had not been recorded in the ExAC Browser previously. The residue T494 was considered as a phosphorylated site according to the PhosphoSitePlus browser (https:// www.phosphosite.org/homeAction.action). The three

Table 2 Laboratory results of the proband

\begin{tabular}{ll}
\hline Test & Results \\
\hline Chromosome karyotype & $46 \mathrm{XX}$, normal \\
Plasma ammonia & normal \\
Lactate & elevated $(2.4-4.7 \mathrm{mmol} / \mathrm{L}$, normal range: less than $2.2 \mathrm{mmol} / \mathrm{L})$ \\
TORCH & negative \\
Screening of genetic metabolism & slightly elevated lactate in blood and urine \\
Electroencephalogram & abnormal (prominent fast wave indicated in frontal region) \\
Auditory brain-stem responses & normal \\
Doppler ultrasound echocardiography & normal \\
Creatinine & elevated $(202 \mu \mathrm{mol} / \mathrm{L}, \mathrm{normal}$ range:45-84 $\mu \mathrm{mol} / \mathrm{L})$ \\
Urea nitrogen & $25.84(2.9-8.2) \mathrm{mmol} / \mathrm{L}$ \\
Uric Acid & $828.4(155-357) \mu \mathrm{mol} / \mathrm{L}$ \\
Thyroid function & normal \\
Glutamic oxalacetic transaminase & $396.7(10-67) \mathrm{U} / \mathrm{L}$ \\
Glutamic pyruvic transaminase & $100.2(5-35) \mathrm{U} / \mathrm{L}$ \\
Bilirubin & normal \\
Cardiac troponin I & $0.08(0.00-0.09) \mathrm{pg} / \mathrm{ml}$ \\
Creatine kinase isoenzyme & $2.5(0.0-3.7) \mathrm{ng} / \mathrm{ml}$ \\
Myoglobin & $77.4(11.6-73.0) \mathrm{ng} / \mathrm{ml}$ \\
Triglyceride & normal \\
Blood glucose & normal $(4.7-7.5 \mathrm{mmo} / \mathrm{L})$ \\
\hline
\end{tabular}

TORCH serum antibody tests for toxoplasma, rubella virus, cytomegalovirus, and herpes simplex virus 

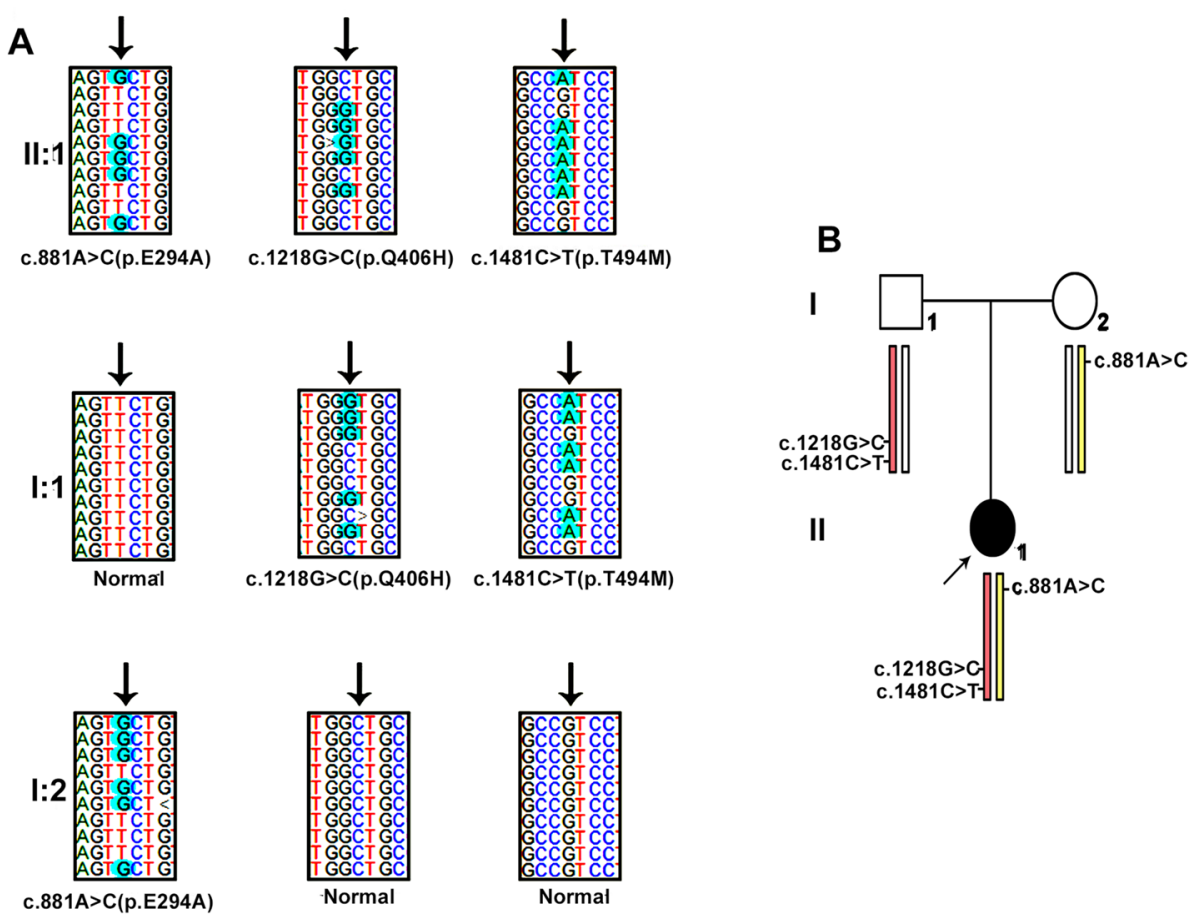

Fig. 2 A The mutations in the pedigree. Three mutations identified in the proband (II:1). Two mutations identified in the father (l:1). One mutation identified in the mother (I:2). B The pedigree information. The proband (I:1) was indicated by the arrow. The parents (I:1, I:2) appeared normal

mutated residues were all evolutionarily well conserved (Fig. 3A), which indicates these residues have important functions.

To explore whether the mutations could lead to protein misfolding, homologous models of truncated wild-type and mutated TRMT5 fragments including each mutated residue were generated. The peptides containing p.E294A and p.Q406H were modeled on SWISS-MODEL (https:// swissmodel.expasy.org/). As SWISS-MODEL could only build the model from residue 96 to 469, we built the peptide model from residue 406 to 509 (which contained p.T494M) on QUARK (https://zhanglab.ccmb.med. umich.edu/QUARK2/). The hydrogen bonds were analyzed by PyMOL (ver 1.4.1). There was one more hydrogen bond between R291 and the mutated residue A294 than between R291 and the wild-type E294 (Fig. 3B and C). Moreover, there were two more hydrogen bonds between the mutated residues H406 and D404 and S410 than between the wild-type Q406 and D404 and S410 (Fig. 3D and E), and there were two hydrogen bonds less between the mutated residues M494 and R491 and F498 than between the wild-type T494 and R491 and F498 (Fig. 3F and G). All of the alterations in hydrogen bonds might lead to misfolding of TRMT5 to affect its normal function. The bioinformatic analysis indicated these three mutations in TRMT5 were all associated with COXPD26.

\section{Discussion and conclusion}

Our case presented recurrent pneumonia, shortness of breath, gastrointestinal dysmotility with frequent vomiting, severe developmental delay, muscular hypotonia and exercise intolerance, and renal and hepatic defects. She was unable to sit or stand without help. In addition, her motor development, speech, and social adaptation were delayed, which is consistent with previous reports [1-3]. Recurrent seizures with spastic diplegia appeared when she was 5 years old, which is consistent with Patient 2 in Mark's article [3]. A wide extracerebral space and thin corpus callosum were indicated by brain MRI. Brain MRI of this disease is not specific, as each patient has different manifestations. Muscle biopsy showed myopathic features without ragged-red fibers, as was the case for the 7-year-old patient in Powell's paper [2]. Muscle biopsy also indicated reduced respiratory chain complex enzyme activities. Unfortunately, the enzyme activity of COX could not be detected in our lab. However, liver pathology of this disease has not been reported in previous research. In the present study, light and electron microscopy indicated a large amount of glycogen and high fat accumulation in the liver, which likely led to liver damage with increased collagen deposition and Kupffer cell activation. Blood glucose levels were within the normal range, but the patient's delayed motor development 


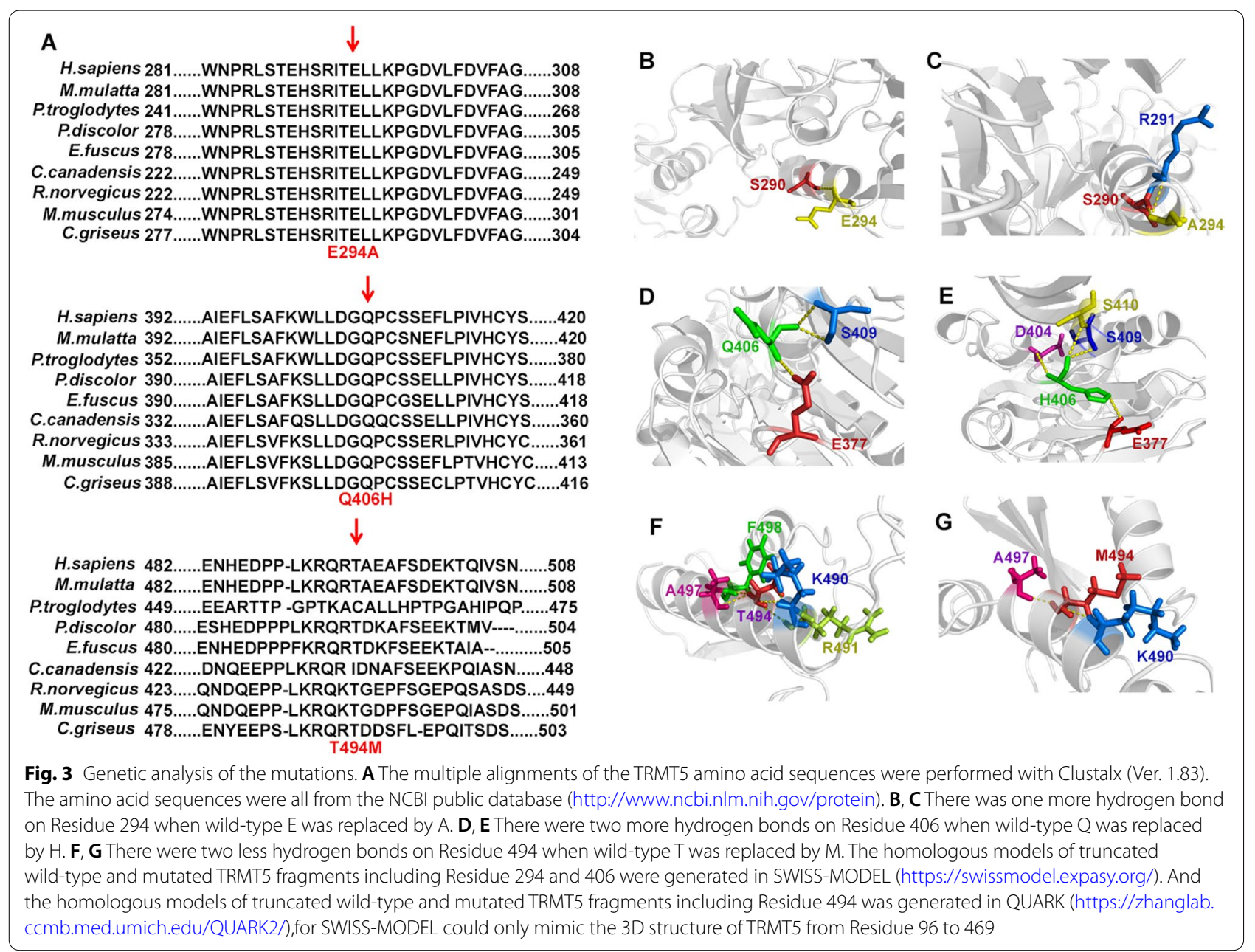

and exercise intolerance indirectly suggested mitochondrial complex deficiency, as previously reported in other cases [1-3], eventually leading to reduced glycogen utilization, followed by the conversion of excessive glycogen into fatty acid, which manifested as increased fatty acid levels in the liver and muscle tissue.

To some extent, our observations indicate novel features of this disease. Most of the clinical features, including early onset, muscle weakness, exercise intolerance, neuropathy, and spastic diplegia, were shared with previously reported patients. The manifestation of developmental delay, lactic acidosis, seizure, and gastrointestinal, renal, and liver problems also overlapped with previous cases [1-3].

There were four COXPD26 patients in previous reports, who all carried two compound heterozygous mutations/deletions in TRMT5. They shared one common deletion, c.312_315del, which leads to a frameshift $[2,3]$. In our study, the proband had different compound heterozygous missense mutations of TRMT5: c.881A $>\mathrm{C}$ (p.E294A) from her mother and c.1218G>C (p.Q406H) and c.1481C $>$ T (p.T494M) from her father. The parents appeared normal, whereas the proband had a severe syndrome and died from stroke and respiratory failure at the age of 5 years. To the best of our knowledge, these are the first TRMT5 mutations identified in Asian COXPD26 patients. All previous COXPD26 patients had early symptom onset, but the patients lived longer than our proband and had a higher quality of life. Our case presented with severe symptoms at an early age and with different manifestations than the other COXPD26 patients, which may be the result of the genotype identified in this patient. Further analysis is necessary to confirm this hypothesis.

In the present study, medical exome sequencing was used to explore the possible genetic defects resulting in the disease. Compared to whole genome and whole exome sequencing, medical exome sequencing focuses on clinical interpretable regions of genes, which greatly improves the accuracy of sequencing and broadens the spectrum [12]. Given that the phenotype of the proband was much more severe than those in previous reports, we considered whether there was molecular "double trouble" 
in the patient's genome. However, we did not find any known copy number variation which was associated with the phenotypes. We excluded any other candidate genes based on our whole exome sequencing results for either of the two reasons.

In conclusion, we identified novel heterozygous mutations of TRMT5 that cause COXPD26 for the first time in a Chinese family. This study will further our understanding of the molecular mechanisms of COXPD26 and contribute to elucidation of the phenotype-genotype correlations of related disorders.

\section{Abbreviations}

COXPD26: Combined oxidative phosphorylation deficiency 26; TRMT5: tRNA (guanine(37)-N1 methyltransferase 5; MRI: Magnetic resonance imaging; ABR: Auditory brain-stem responses; MAF: Minor-allele frequency; CNV: Copy number variation.

\section{Acknowledgements}

We thank International Science Editing (http://www.internationalscienceedit ng.com) for editing this manuscript.

\section{Authors' contributions}

SW: Designed the research, collected clinical data, analyzed the data and drafted the manuscript; WL and ZB: Participated in analyzing the part of data; $\mathrm{HC}$ and YL1: Participated in the communicate with patients' guardians; SH and DY: Collected clinical data; YL2:Designed the research, analyzed the data and drafted part of the manuscript; HL: Participated to the in discussion and interpretation of the data and results, involved in the critical revision of this manuscript and take the primary responsibility of this research; All authors have read and approved this manuscript and ensure that this is the case.

\section{Funding}

Design of the study and collection, analysis, and interpretation of data and in writing the manuscript were funded by the National Natural Science Foundation of China (No. 81803116), the Basic Research Project for Medical and Health Applications of Suzhou City (SYS2019083) and Natural Science Foundation of Jiangsu Province (BK20210097 and BK20211077).

\section{Availability of data and materials}

The datasets used and/or analysed during the current study are available from the corresponding author (Haitao Lv and Ying Liu) on reasonable request.

\section{Declarations}

\section{Ethics approval and consent to participate}

Ethical approval for this study was obtained from the local ethics committee (Children's Hospital of Soochow University, No. 2019LW010). Written informed consent was obtained from the patient's parents.

\section{Consent for publication}

The guardians gave their written consent for publication and the proof of consent can be requested at any time.

\section{Competing interests}

The authors declare that they have no conflict of interest.

\section{Author details}

${ }^{1}$ Pediatric Intensive Care Unit, Children's Hospital of Soochow University, Suzhou, Jiangsu Province 215025, P.R. China. ${ }^{2}$ Institute of Pediatric Research, Children's Hospital of Soochow University, Suzhou, Jiangsu Province 215025, P.R. China. ${ }^{3}$ Department of Cardiology, Children's Hospital of Soochow University, Suzhou, Jiangsu Province 215025, P.R. China.
Received: 9 August 2021 Accepted: 27 January 2022

Published online: 02 February 2022

\section{References}

1. Haller RG, Lewis SF, Estabrook RW, DiMauro S, Servidei S, Foster DW. Exercise intolerance, lactic acidosis, and abnormal cardiopulmonary regulation in exercise associated with adult skeletal muscle cytochrome c oxidase deficiency. J. Clin. Invest. 1989;84:155-61.

2. Powell CA, Kopajtich R, D'Souza AR, Rorbach J, Kremer LS, Husain RA, et al TRMT5 mutations cause a defect in post-transcriptional modification of mitochondrial tRNA associated with multiple respiratory-chain deficiencies. Am. J. Hum. Genet. 2015;97(2):319-28.

3. Tarnopolsky MA, Brady L, Tetreault M. Care4Rare Canada Consortium: TRMT5 mutations are associated with features of complex hereditary spastic paraparesis. Neurology. 2017;89(21):2210-1.

4. Christian T, Gamper H, Hou YM. Conservation of structure and mechanism by Trm5 enzymes. RNA. 2013;19(9):1192-9.

5. Sakaguchi R, Giessing A, Dai Q, Lahoud G, Liutkeviciute Z, Klimasauskas $\mathrm{S}$, et al. Recognition of guanosine by dissimilar tRNA methyltransferases. RNA. 2012;18(9):1687-701.

6. Goto-ito S, Ito T, Ishii R, Muto Y, Bessho Y, Yokoyama S. Crystal structure of archaeal tRNA(m1G37) methyltransferase aTrm5. Proteins. 2008;72(4):1274-89.

7. Lahoud G, Goto-Ito S, Yoshida K, Ito T, Yokoyama S, Hou YM. Differentiating analogous tRNA methyltransferases by fragments of the methyl donor. RNA. 2011;17(7):1236-46.

8. Hou YM, Masuda I. Kinetic Analysis of tRNA Methyltransferases. Methods Enzymol. 2015;560:91-116.

9. Brulé H, Elliott M, Redlak M, Zehner ZE, Holmes WM. Isolation and characterization of the human tRNA-(N1G37) methyltransferase (TRM5) and comparison to the Escherichia coli TrmD protein. Biochemistry. 2004;43(28):9243-55

10. Baba T, Ara T, Hasegawa M, Takai Y, Okumura Y, Baba M, et al. Construction of Escherichia Coli K-12 in-frame, single gene knockout mutants: the Keio collection. Mol Syst Biol. 2006;2006(2):0008.

11. Anderson S, Bankier AT, Barrell BG, de Bruijn MH, Coulson AR, Drouin J, et al. Sequence and organization of the human mitochondrial genome. Nature. 1981;290(5806):457-65.

12. Wu S, Bai Z, Dong X, Yang D, Chen H, Hua J, et al. Novel mutations of the POLR3A gene caused POLR3-related leukodystrophy in a Chinese family: a case report. BMC Pediatr. 2019;19(1):289.

\section{Publisher's Note}

Springer Nature remains neutral with regard to jurisdictional claims in published maps and institutional affiliations.
Ready to submit your research? Choose BMC and benefit from:

- fast, convenient online submission

- thorough peer review by experienced researchers in your field

- rapid publication on acceptance

- support for research data, including large and complex data types

- gold Open Access which fosters wider collaboration and increased citations

- maximum visibility for your research: over $100 \mathrm{M}$ website views per year

At $\mathrm{BMC}$, research is always in progress.

Learn more biomedcentral.com/submissions 